time course or remain open only while the button is depressed. In one experiment on similarity scaling of frequency-modulated tones, the stimulus generation and control equipment consisted of two voltage-controlled generators, voltage dividers, and an electronic switch. For another experiment on voice similarity, the apparatus will be connected to a small computer with a rapid random-access disk. Monosyllabic utterances, stored in digital form on the disk, will be retrieved and acoustically reproduced at the touch of a button.

At present, E must visually observe and record the configuration of buttons at the end of each trial. The recording function could be rendered automatic by adding a slide wire potentiometer to the back of each bar and connecting these potentiometers to voltage sources and measuring equipment. This equipment would record a button's position at each depression as well as the durations and sequence of button pushings (i.e., stimulus presentations). In this last respect, auditory stimulus sorting can furnish more potentially useful information than the sorting of manipulable visual stimuli, which does not yield precise information about S's experimental exposure to each stimulus.

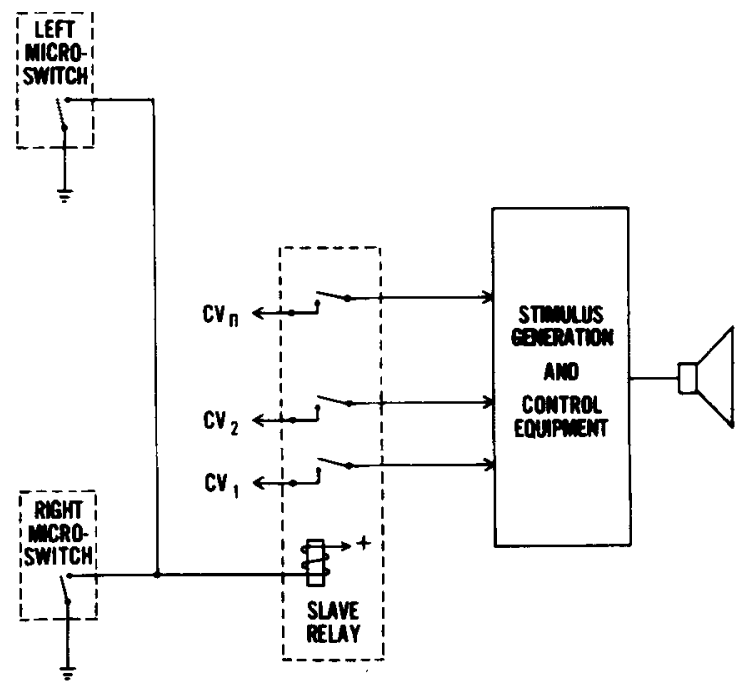

Fig. 3. Block diagram showing connection of sorting apparatus to stimulus generator.

\title{
Simultaneous switching of auditory transients for two-channel presentation'
}

\author{
RICHARD T. TOMSIC and LLOYD F. ELFNER, FLORIDA \\ STATE UNIVERSITY, Tallahassee, Fla. 32306
}

A modification of the Grason-Stadler Series $829 D \& E$ Electronic Switches that enables simultaneous cross-channel presentation of auditory signals at the two ears is described.

The human auditory system is extremely sensitive to interaural differences in arrival time of signals. A dichotically presented signal having an interaural arrival time discrepancy on the order of microseconds, is discriminable from a simultaneously presented signal. Therefore, it is imperative to exercise precise control of the onset and offset times. One of the most commonly used instruments employed in this area is the Grason-Stadler Series 829 (D or E) Electronic Switch. Even though the two channels of the switch are regulated by the same signal, slight differences in electronic tube characteristics can cause one channel to start conducting before the other. When two channels of the switch are used to turn on and turn off auditory signals simultaneously (within certain limits) at the two ears, the difference in the onset of the auditory signals is detectable.

A major portion of the difference in onset and offset times of the switched signal can be eliminated by matching the $6 \mathrm{SN} 7$ modulator tubes between the two channels (V51, V71, V52, and V72). Since tubes within each channel should be matched to minimize switching transients the task becomes one of matching four tubes. A potentiometer is supplied within each channel to compensate for tube differences to minimize switching transients but the same is not true for cross-channel matching.

In the unmodified circuit (Fig. 1a) that connects the biasing voltage or switching signal to the modulator tubes, the signal is passed through two $10 \mathrm{~K}$ resistors (R 112 and R 113, in the A switching circuits), one to each channel. In the modified circuit
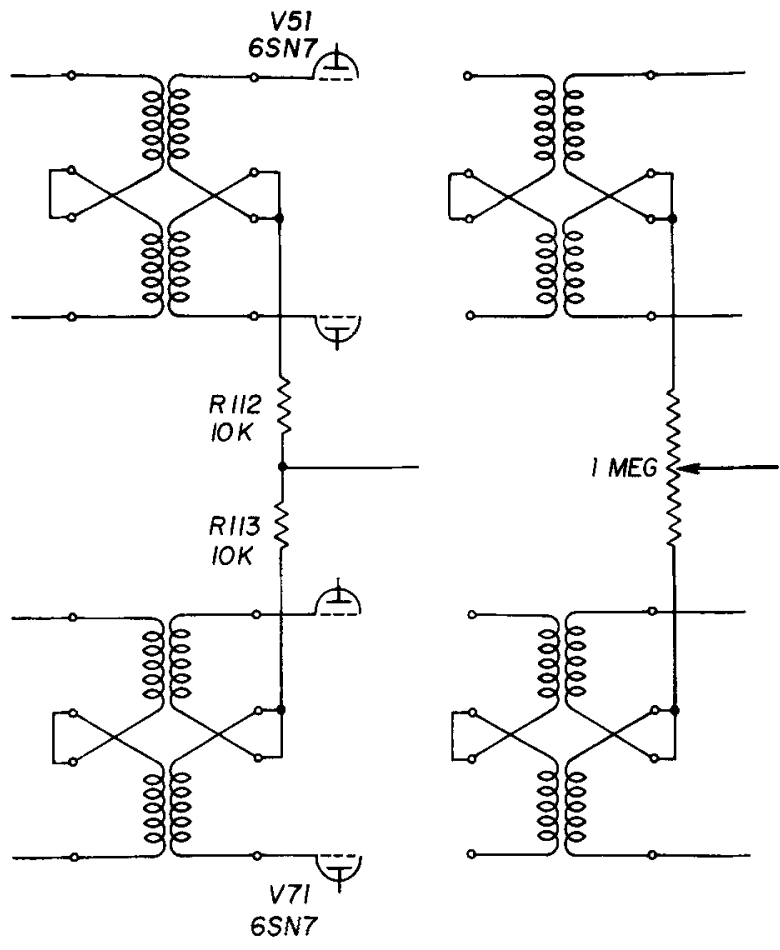

o. UNMODIFIED CIRCUIT

b. MODIFIED CIRCUIT 
(Fig. 1b) a 1-megohm potentiometer replaces the two $10 \mathrm{~K}$ resistors with the center tap connected to the bias voltage clamping circuit. The potentiometer allows the adjustment of the bias vol tage to the modulator tubes.

While the tubes are resting in either the steady ON state or steady OFF state, the bias voltages are equal regardless of the potentiometer setting since virtually no current is passing through the potentiometer. During the transient portions of the switching operation when the bias voltages on the tubes are changing, there is a slight current and the difference in resistance to the two channels, as determined by the potentiometer setting, will have the effect of leading one tube ahead of the other.

The potentiometer can also be inserted in place of the two resistors R 114 and R 115 (see Grason-Stadler operating manual), in the B switching circuits. With the two potentiometers the two channels can be brought into close coordination. If the modulator tubes are sufficiently similar in characteristics the two channels can be made to switch virtually simultaneously. If the tubes are not sufficiently similar to allow elimination of the onset and offset differences, the potentiometers can be adjusted so that onset difference is nil and the offset difference is at a minimum.

Another modification can be made to adjust for simultaneous switching of two switches that are triggered by the same timer. The $30 \mathrm{~K}$ resistor (R27) that determines the plate voltage on $V_{4}$ and $V_{5}$ can be replaced with a $22 \mathrm{~K}$ resistor and a $10 \mathrm{~K}$ potentiometer as shown in Fig. 2. This procedure allows for regulation of the voltage of the clamping circuit, hence, the time at which the modulator tubes will be turned on. The best way to check for differences in switching onset and offset disparities is by a differential amplifier on an oscilloscope. A low remainder voltage indicates a balanced system.

The potentiometers can be mounted on the rear of the chassis behind the Channel 1 switching section.

\section{NOTE}

1. This study was supported by NSF Grant GB-6815 and NIH Grant MH 11218.
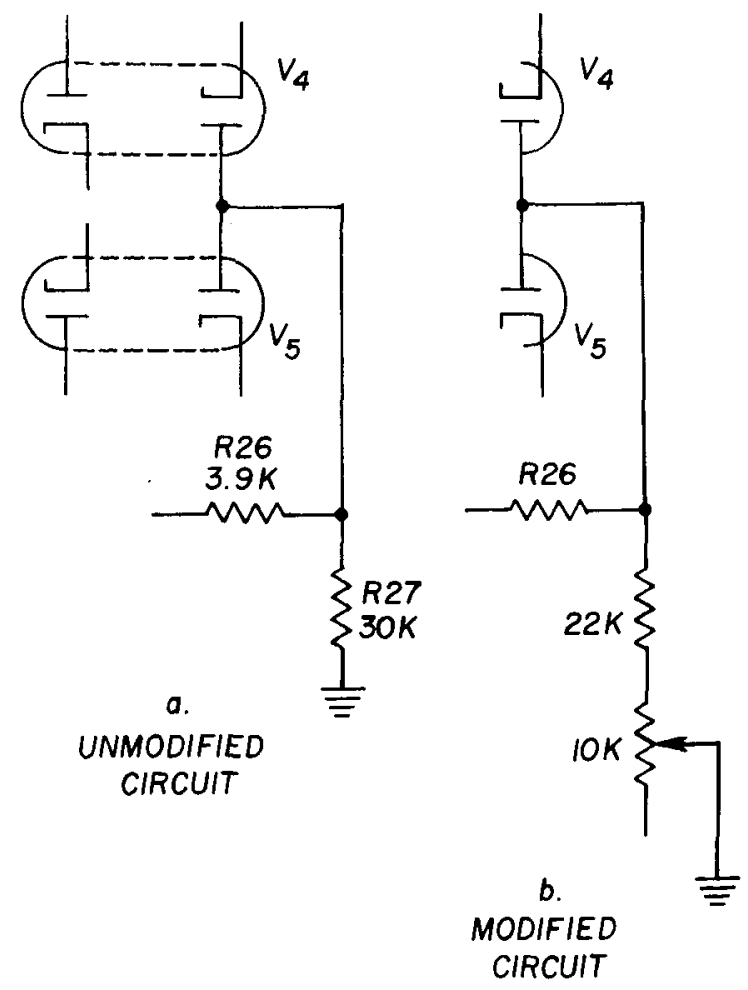

Fig. 2. Schematic for adjusting overall switching tube bias.

\section{A simple method of producing discrete subcortical lesions using radio-frequency currents ${ }^{1,2}$}

\author{
T. E. LeVERE and LAWRENCE R. NICHOLSON, NORTH \\ CAROLINA STATE UNIVERSITY, Raleigh, North Carolina \\ 27607
}

The described procedures represent an evaluation of the use of radio frequency currents in the production of discrete subcortical brain lesions. The methods described and the electrodes used indicated that RF lesion size was closely and linearly related to a simple $V d c$ index and that the resultant lesions were equally distributed around the position of the lesion electrode with extremely smooth and consistent perineters.

The utility of a reliable method for destroying discrete areas of neural tissue below the surface of the brain can be estimated from the varied techniques and equipment that have been devised for such operative procedures. By and large, the use of unidirectional current concentrated at the tip of an electrode stereotaxically located within the brain has enjoyed the most success. This electrolytic technique requires simple equipment and provides close correlation of lesion size with millicoulomb flow (Macintyre et al, 1957). The production of subcortical brain lesions, however, with dc currents is not without its drawbacks. Perhaps the most serious effects are the inconsistency of lesion shape and the deposition of metallic ions at the perimeter of the insult, with the subsequent possibility of stimulation of adjacent brain areas (Reynolds, 1963).

An alternative technique for producing subcortical lesions utilizes bidirectional radio-frequency $(\mathrm{RF})$ currents that, when concentrated at the tip of an electrode, can cause neural destruction by the conversion of electrical energy into heat (Aronow, 1960; Reynolds, 1963). This method has been used in our laboratory and the results are described herein. Specifically, lesion size was studied as a function of electrode configuration and power output.

\section{SUBJECTS}

The Ss were four male and nine female adult Long-Evans hooded rats from the colony maintained at North Carolina State University. All Ss were in good health and treated in accord with accepted practices concerning experimental animal surgery.

\section{APPARATUS}

A Grass Model LM-3 RF lesion generator was used for all experimental lesions. The electrodes were constructed from either 